\title{
Which intraocular lens would ophthalmologists choose for themselves?
}

\author{
Hercules D. Logothetis ${ }^{1} \cdot$ Robert S. Feder $^{1}$
}

Received: 10 May 2018 / Revised: 10 March 2019 / Accepted: 29 April 2019 / Published online: 14 May 2019

(c) The Royal College of Ophthalmologists 2019

\begin{abstract}
Purpose Contemporary cataract surgery is increasingly customizable with the advent of presbyopia-correcting intraocular lenses (IOLs) and the femtosecond laser. The purpose of this study was to determine the types of IOLs ophthalmologists choose for themselves and whether demographic characteristics, surgical experience, and attitudes of ophthalmologists might influence their decision-making. Additional goals included evaluating the use of femtosecond laser use in cataract surgery and investigating surgeon IOL preferences for their patients.

Methods We distributed a 29-question Survey Monkey survey to senior ophthalmology residents and practicing ophthalmologists and received 347 responses during a 1-month period. We analyzed 328 surveys using chi-square tests and Fisher's exact tests.

Results Main outcome measures included surgeons' personal preferences for choice of IOL, femtosecond laser-assisted cataract surgery, and IOL preference for patients. In the setting of no astigmatism, $61.3 \%$ of respondents would choose a monofocal IOL set for either distance or monovision for their own surgery. For corneal astigmatism $>1.25 \mathrm{D}, 60.3 \%$ of respondents would choose a toric monofocal lens and only $6.9 \%$ would want a femtosecond laser or manual LRI for their own surgery. Of the respondents, $34.6 \%$ perform femtosecond laser-assisted cataract surgery, but only $15.3 \%$ would want femtosecond cataract surgery performed on themselves. Also, 67.7\% implant presbyopia-correcting IOLs (diffractive echelette, multifocal, or accommodative). When correcting for patients' corneal astigmatism $>1.25 \mathrm{D}, 65.7 \%$ of respondents preferred a toric monofocal IOL.

Conclusion Ophthalmologists value quality of vision and are risk averse. Overall, a surgeons' personal preference for their own surgery is correlated with past surgical experience with patients.
\end{abstract}

\section{Background}

Cataract surgery is one of the most common operations performed in the United States. Continued advances in technology have resulted in various surgical options available for the cataract patient, many of which require an

Meeting presentation: This work was presented at Northwestern Ophthalmology and Vision Research Day, Chicago, IL, USA on 30 June 2017 and also at the American Academy of Ophthalmology Annual Meeting, New Orleans, LA, USA on 12 November 2017

Hercules D. Logothetis

herculeslogothetis@gmail.com

1 Department of Ophthalmology, Northwestern University, Fienberg School of Medicine, 645 N. Michigan Avenue, Suite 440, Chicago, IL 60611, USA additional out-of-pocket expense. Multiple intraocular lens (IOL) options exist with the promise of reducing corneal astigmatism and/or dependence on glasses for distance and reading. Additionally, the use of femtosecond laser in cataract surgery has offered an additional option for surgeons to offer their cataract surgery patients. Patient goals vary and require a customized approach for cataract surgery planning. There are currently no studies evaluating the IOL and femtosecond laser preferences that cataract surgeons would choose for themselves. It has been suggested that surgeons' decision with regard to cataract surgery may vary depending on whether the surgery is for themselves or for their patients [1].

Current literature regarding the utility of femtosecond laser-assisted cataract surgery (FLACS) is conflicting. Studies show both benefits and drawbacks to the use of this laser approach to cataract surgery. FLACS improves precision and reproducibility of corneal incisions and capsular 
opening and may reduce the amount of ultrasound energy ultimately required for lens nucleus emulsification [2]. It is not clear, however, if these statistically significant differences result in meaningful improvement in postoperative visual outcomes. FLACS requires longer overall procedure times compared to phacoemulsification even after 200 cases $[3,4]$. Comparison of multifocal IOL to accommodative IOL in studies yields a wide range of results. One study found multifocal and accommodative IOL equal for intermediate and distance but multifocal superior at near [5]. Another study found the accommodative 1OL Crystalens (Bausch and Lomb, Inc., Rochester, NY, USA) better when compared to ReSTOR (Alcon Laboratories, Inc., Fort Worth, TX, USA) and Tecnis 4.0 (Tecnis Abbott Medical Optics, Inc., Santa Ana, CA, USA) for uncorrected distance visual acuity but not for corrected distance visual acuity. That same study found a multifocal IOL better at near [6]. A study looking at resident success with multifocal IOL and toric lenses found ophthalmology residents' patients were more likely to have better uncorrected distance visual acuity with multifocal lenses compared to toric lenses [4]. Newer technologies such as the toric multifocal IOL have presented a newer option that was found to be safe and effective as well [7]. More recently, many publications have reviewed the results of the Tecnis Symfony IOL (Abbott Optics, Santa Ana, CA, USA). Early results are demonstrating high patient satisfaction throughout all ranges of vision [8].

This study provides insight into how cataract surgeons weigh different variables when deciding on their own surgery in the context of personal characteristics and experience. We evaluated the surgical preferences of practicing ophthalmologists and PGY-4 ophthalmology residents with regard to cataract surgery proposed for themselves. We identified characteristics and trends that might be associated with the selection of various IOL options and strategies.

\section{Methods}

\section{Survey}

The survey was comprised of 29 questions. It was subdivided into sections, which consisted of consent, demographics, desired goals of surgery, IOL selection, and experience with presbyopia-correcting IOL exchange. Question formats included multiple choice, Likert scales, and open-ended text or numerically based answers. The survey was created, completed, and submitted on SurveyMonkey.com (San Mateo, CA). The majority of complete surveys took respondents approximately $5 \mathrm{~min}$ to complete.

\section{Participants and collection}

The AUPO listserv, with permission from the AUPO, was utilized to distribute the SurveyMonkey link to the program directors of all existing ACGME accredited ophthalmology residency programs. Approximately $10-15$ of the listed email addresses were returned to sender marked as erroneous addresses while the remaining 100 email addresses successfully arrived at the listed addresses. The survey was also sent to the director of each state ophthalmology society for distribution to their membership. The survey was sent to the department chair of each academic ophthalmology program in Chicago for distribution to the department members. Finally, the survey was sent to members of the Cornea Society. Survey submissions were collected between 16 January 2017 and 13 February 2017. A total of 347 surveys were completed of which 328 were analyzed. Inclusion criteria for analysis required completion of the IRB-approved survey and the consent. Participation in the study was voluntary, responses were confidential, data were submitted anonymously, and no incentives were provided for participating. The authors sent one follow-up email to initial contacts over the 1-month period the survey was open for submissions to encourage participation. No repeat submissions were permitted from the same IP address.

\section{Statistical analysis}

Survey responses were individually transferred from SurveyMonkey.com into a Microsoft Excel file. Descriptive summary statistics were completed on the SAS software (SAS Institute Inc. 2012. SAS OnlineDoc 9.4. Cary, NC: SAS Institute Inc.). We used chi-square tests, rank-sum tests, and logistic regression for multivariate analysis. All tests were two sided. There were no adjustments for multiple statistical testing.

\section{Results}

\section{Demographics}

Of the 328 survey respondents (Table 1a), the average age was $50.3( \pm 12.8)$ years. Approximately $67 \%$ of those surveyed had been in practice for $>10$ years. Practice settings were full-time academic practice $(36.6 \%)$, multi-specialty private practice $(26.8 \%)$, small group practice of $<3$ physicians $(18.0 \%)$, and solo practice $(10.1 \%)$. Most of the respondents (Table $1 \mathrm{~b}$ ) were myopic $(37.3 \%)$ or had myopic astigmatism $(35.5 \%)$ while $9.0 \%$ were hyperopic or hyperopic with astigmatism and $10.1 \%$ did not report having refractive error. More than half $(63.9 \%)$ of the respondents were presbyopic. Of the ophthalmologists surveyed, 
Table 1a Demographics

\begin{tabular}{ll}
\hline Age & \\
$N$ & 328 \\
Mean & 50.3 \\
Standard deviation & 12.8 \\
Median & 51 \\
Range & $27-85$ \\
Experience, $N(\%)$ & \\
$<10$ years in practice & $107(32.6 \%)$ \\
$>10$ years in practice & $221(67.4 \%)$ \\
Practice, $N$ (\%) & \\
Full-time academic practice & $120(36.6 \%)$ \\
Multi-specialty private practice & $88(26.8 \%)$ \\
Small group practice $(<3$ physicians $)$ & $59(18.0 \%)$ \\
Solo practice & $33(10.1 \%)$ \\
None of the above & $20(6.1 \%)$ \\
Unknown & $8(2.4 \%)$ \\
\hline
\end{tabular}

Table 1b Demographics

\begin{tabular}{|c|c|c|}
\hline & $N$ & Percentage \\
\hline \multicolumn{3}{|l|}{ Type of refractive error } \\
\hline Myopia & 103 & $37.3 \%$ \\
\hline Myopic astigmatism & 98 & $35.5 \%$ \\
\hline Hyperopia & 15 & $5.4 \%$ \\
\hline Hyperopic astigmatism & 10 & $3.6 \%$ \\
\hline Mixed astigmatism & 8 & $2.9 \%$ \\
\hline No refractive error & 28 & $10.1 \%$ \\
\hline Other & 14 & $5.1 \%$ \\
\hline \multicolumn{3}{|l|}{ Presbyopia } \\
\hline No & 118 & $36.1 \%$ \\
\hline Yes & 209 & $63.9 \%$ \\
\hline \multicolumn{3}{|l|}{ Performed cataract surgery } \\
\hline Yes & 274 & $85.5 \%$ \\
\hline No & 54 & $16.5 \%$ \\
\hline \multicolumn{3}{|c|}{ Number of cataract surgeries performed monthly } \\
\hline$<10$ & 37 & $11.4 \%$ \\
\hline $10-20$ & 52 & $16.0 \%$ \\
\hline$>20$ & 189 & $58.2 \%$ \\
\hline Did not perform cataract surgery & 47 & $14.5 \%$ \\
\hline \multicolumn{3}{|l|}{ Implant premium IOLs } \\
\hline Yes & 222 & $67.7 \%$ \\
\hline No & 106 & $32.3 \%$ \\
\hline
\end{tabular}

$I O L$ intraocular lens

$85.5 \%$ perform cataract surgery and $67.7 \%$ of them implant presbyopia-correcting IOLs (diffractive echelette, multifocal, or accommodative). Of the respondents, $58.2 \%$ performed $>20$ cataract surgeries per month, while the others performed $<20$ or none at all.

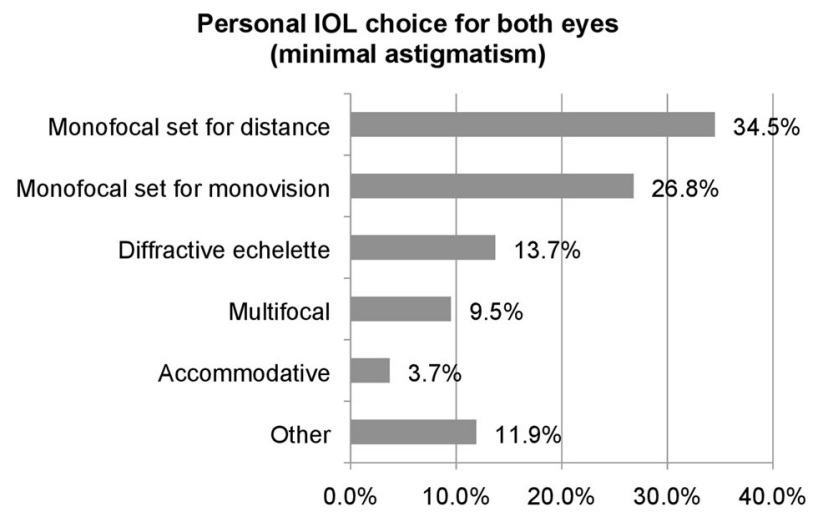

Fig. 1 Surgeon personal intraocular lens choice for both eyes assuming minimal astigmatism. Bar graph showing which option surgeons would choose for their own surgery assuming they had minimal astigmatism

\section{Ophthalmologists' attitudes}

The response to the question "How do you rate yourself with regard to risk?", was recorded in a Likert scale format. Respondents were provided answer choices 1 (risk averse) to 5 (risk taker). For the purpose of data analysis, the responses 1 (risk averse) and 2 (between risk averse and neutral) were combined into 1 category labeled risk averse. The response option 3 was left as neutral. The responses 4 (between neutral and risk taker) and 5 (risk taker) were combined and labeled risk taker. Over half of respondents $(50.9 \%)$ perceived themselves as risk averse, while only $18.0 \%$ considered themselves risk takers. The remaining $31.1 \%$ responded neutral.

Respondents were asked how they value quality of vision in the context of their own cataract surgery. Once again, a Likert scale 1-5 with 1 indicating least important and 5 indicating most important was used to assess respondents' opinions. An overwhelming number of respondents $(93.0 \%)$ rated quality of vision to be most important, response 5. No respondents replied with options 1 or 2 , indicating less importance. The remaining $7.0 \%$ chose options 3 or 4 .

Lastly, respondents were asked how they value glasses independence in the context of their own cataract surgery. A Likert scale format with 1 indicating not important and 5 indicating most important was used to assess respondents' opinions. Similar to Fig. 1, responses 1 and 2 were combined into one category labeled "least important" and responses 4 and 5 were combined into a category labeled "most important". Responses varied among the three response categories. Of the responding ophthalmologists, $25.4 \%$ rated glasses independence as the least important while $43.1 \%$ rated glasses independence as the most important. 


\section{Surgeon IOL choice}

A primary goal of our study was to identify surgeons' IOL preferences for cataract surgery if done on themselves. Figure 1 demonstrates surgeons' IOL choice assuming there was minimal astigmatism. Monofocal set for distance was selected by $34.5 \%$ of respondents. The second highest response was monofocal IOL intended for monovision at 26.8\%. The two monofocal options combined made up $61.3 \%$ of responses. The choices for presbyopia-correcting IOLs were the diffractive echelette IOL (Symfony, Johnson and Johnson, Santa Ana, CA, USA) $13.7 \%$, multifocal IOL 9.5\%, and accommodative IOL $3.7 \%$. Figure 2 demonstrates similar data but in the setting of $>1.25 \mathrm{D}$ of corneal astigmatism. The majority of respondents opted for toric monofocal $(60.3 \%)$ for their own surgery. The diffractive echelette toric IOL had a response rate of $14.0 \%$ while the remaining options were all $<10 \%$.

When taking into account presbyopia status, in the setting of minimal astigmatism, $60.8 \%$ of presbyopes would select a monofocal lens for distance or monovision while $24.0 \%$ would choose a presbyopia-correcting lens (diffractive echelette, multifocal, accommodative). Similarly, $61.9 \%$ of non-presbyopes would choose a monofocal lens for distance or monovision while $32.2 \%$ would choose a presbyopia-correcting lens.

In the setting of $>1.25 \mathrm{D}$ of astigmatism, $73.0 \%$ of presbyopes would choose a monofocal or toric monofocal lens while $20.0 \%$ would choose an alternative option (accommodative toric, diffractive echelette toric, or limbal relaxing incision (LRI)). Similarly, $65.8 \%$ of nonpresbyopes would choose a monofocal or toric monofocal lens while $29.9 \%$ would choose one of the alternatives.

The number of participants that identified as hyperopes was very small making analysis of IOL selection with regards to refractive error statistically insignificant.

\section{Personal IOL choice for both eyes (>1.25D astigmatism)}

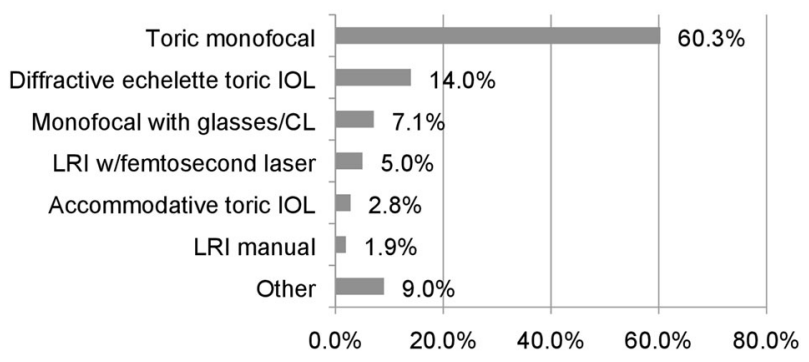

Fig. 2 Surgeon personal intraocular lens (IOL) choice assuming corneal astigmatism $>1.25 \mathrm{D}$. Bar graphs showing which IOL option surgeons choose for themselves assuming they had $>1.25 \mathrm{D}$ of corneal astigmatism
Influence of attitudes and demographics on IOL selection

The main thrust of our data analysis explored the influence of attitudes and demographics on IOL selection in the context of minimal astigmatism and corneal astigmatism $>1.25 \mathrm{D}$. The variables studied in a multivariate analysis included: age, preference for glasses independence, attitude toward risk taking, whether one performs cataract surgery, use of presbyopia-correcting IOLs (diffractive echelette, multifocal, or accommodative), use of FLACS, years in practice, number of multifocal IOL implanted, and number of accommodative IOL implanted. Within the analysis, surgeon IOL choices were divided into two categories. In the setting of minimal preoperative corneal astigmatism, monofocal for distance and monovision were included in one group while the remaining options (diffractive echelette, multifocal, accommodative, or other) comprised a second group.

In the setting of minimal corneal astigmatism, statistically significant correlations were found between surgeon IOL choice and the number of multifocal IOL inserted $(p=0.0049)$, number of accommodative IOLs inserted $(p=0.045)$, and preference for glasses independence $(p=$ 0.003). Of the surgeons who had inserted $<50$ multifocal IOLs, $71.8 \%$ selected a monofocal IOL adjusted for distance or monovision as their personal preference. Of surgeons who had implanted at least 50 multifocal IOLs, $54.6 \%$ would select a presbyopia-correcting IOL for themselves. Surgeons who implanted at least 50 multifocal IOL are two times more likely to select a presbyopiacorrecting IOL option (odds ratio $=2.1[1.26-3.65]$ ). Of the surgeons who implanted $<50$ accommodative IOLs, $65.4 \%$ would choose a monofocal set for distance or monovision while $65.3 \%$ of surgeons who implanted $\geq 50$ accommodative IOLs would choose a presbyopiacorrecting IOL for themselves. Surgeons who implanted at least 50 accommodative IOLs are two times more likely to select a presbyopia-correcting IOL option (odds ratio $=$ 2.0 [1.02-4.15]). Of the surgeons who value glasses independence, $55.0 \%$ would select a presbyopia-correcting IOL for themselves. Of the respondents who did not find glasses independence valuable, $72.3 \%$ would choose a monofocal set for distance or monovision. Surgeons who value glasses independence are two times more likely to select a presbyopia-correcting IOL (odds ratio 2.1 [1.13-4.00]).

In the setting of $>1.25 \mathrm{D}$ of corneal astigmatism, the options of monofocal IOL with astigmatism correction using glasses or contact lenses and toric monofocal were included in one group while the remaining choices (LRI with femtosecond laser, manual LRI, diffractive echelette toric, accommodative toric, or other) were included in a 
second group labeled presbyopia correcting. Correlations were found between IOL choice and number of accommodative IOLs inserted $(p<0.001)$ and between IOL choice and preference for glasses independence $(p<0.001)$. Of the surgeons who implanted $<50$ accommodative IOLs, $65.4 \%$ would choose a monofocal set for distance or monovision/ toric monofocal. Of surgeons who implanted $\geq 50$ accommodative IOLs, $61.2 \%$ would choose a presbyopiacorrecting IOL for themselves. Surgeons who implanted at least 50 accommodative IOLs are over three times more likely to select a presbyopia-correcting IOL option (odds ratio $=3.56[1.79-7.09])$. Of the surgeons who value glasses independence, $48.9 \%$ would select a presbyopiacorrecting IOL for themselves. Of the respondents who did not find glasses independence valuable, $82.7 \%$ would choose a monofocal set for distance or monovision/toric monofocal. Surgeons who value glasses independence are four times more likely to select a presbyopia-correcting IOL (odds ratio $4.36[2.15-8.85]$ ).

\section{Femtosecond laser use}

Data showed that $55.4 \%$ of ophthalmologists surveyed are not certified to use the femtosecond laser to assist in cataract surgery. Of the respondents, $19.3 \%$ are using the femtosecond laser at least one time per week; $11.1 \%$ are using FLACS one time a month and $14.1 \%$ are certified but not using FLACS. In response to the question "If you needed cataract surgery, would you want FLACS?", ophthalmologists responded yes in $15.6 \%$ of cases, may be in $31.5 \%$ of cases, and $52.9 \%$ responded no. Table 2 compares surgeons' use of the femtosecond laser for patient cataract surgery with the preference to have it used for their own surgery. Of the surgeons who utilize the femtosecond laser more than one time per week $55.9 \%$ would choose to have the femtosecond laser used for their surgery. In contrast, $<4 \%$ of those who were not certified to use the femtosecond laser would choose to have it used for their own surgery $(p<0.0001)$.

Table 2 "Would you want femtosecond laser-assisted cataract surgery?" compared with "How often do you use FLACS on your patients?"

\begin{tabular}{lllll}
\hline & Total & Yes & May be & No \\
\hline Not certified & $55.4 \%$ & $3.5 \%$ & $31.4 \%$ & $65.1 \%$ \\
$1 \times /$ month or less & $11.1 \%$ & $14.7 \%$ & $44.1 \%$ & $41.2 \%$ \\
$1 \times /$ week or more & $19.3 \%$ & $55.9 \%$ & $23.7 \%$ & $20.3 \%$ \\
Certified but not using & $14.1 \%$ & $9.3 \%$ & $23.3 \%$ & $67.4 \%$ \\
Total & $100.0 \%$ & & & \\
\hline
\end{tabular}

$N=305$

FLACS femtosecond laser-assisted cataract surgery

$p<0.0001$

\section{IOL preferences for patients}

Although this study was not structured to determine whether surgeons choose the same IOL for themselves as they do for their patients, we did investigate which presbyopiacorrecting IOL options surgeons prefer for their patients as well as how they prefer to treat their patients' astigmatism. The most popular presbyopia-correcting IOL choice for patients was the multifocal IOL (36.4\%). This was followed closely by the diffractive echelette IOL $(30.3 \%)$. The accommodative IOL $(6.5 \%)$ was a distant third choice. Of the respondents to this question, $16.0 \%$ said they do not perform cataract surgery and $10.9 \%$ chose "other" as their choice for patients when using presbyopia-correcting IOL. Figure 3 demonstrates how surgeons prefer to treat their patients with corneal astigmatism $>1.25 \mathrm{D}$. The most popular method is using a toric monofocal IOL (65.7\%). The remaining options (placing wounds in axis of astigmatism, monofocal IOL with glasses correction, monofocal IOL with femtosecond laser LRIs, monofocal IOL with manual LRIs, toric accommodative IOL, and other) were chosen with less frequency. Of the respondents to this question, $13.7 \%$ indicated that they do not perform cataract surgery.

\section{Discussion and conclusions}

The goal of this study was to determine IOL preferences of contemporary ophthalmologists, characterize current femtosecond laser use, and evaluate presbyopia-correcting IOL preferences used for patients. It was also of interest to characterize surgeons' attitudes toward risk taking, quality of vision, and glasses independence. The data collected were from ophthalmologists of all ages and practice settings. Of the greatest interest was whether surgeons' personal choices were influenced by demographics, experience, and attitudes.

It is common for a preoperative cataract consultation to include questions regarding the importance of quality of vision and the importance of glasses independence. It is

\section{IOL Choice for PATIENTS (>1.25D astigmatism)}

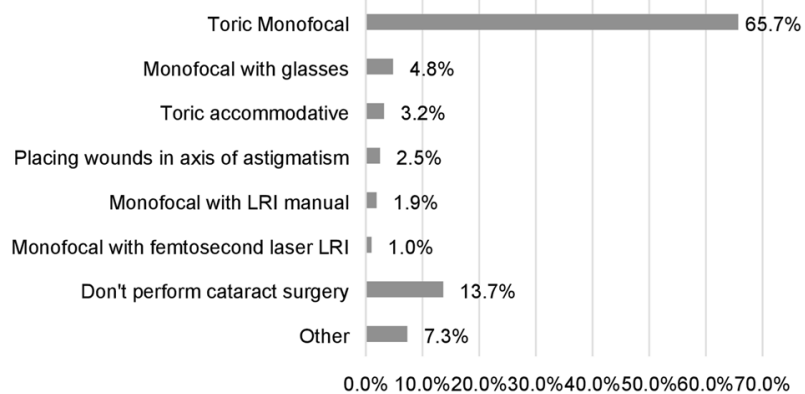

Fig. 3 Bar graph showing surgeon intraocular lens choice for patients with corneal astigmatism $>1.25 \mathrm{D}$ 
perhaps not surprising that $98.8 \%$ of eye surgeons rated quality of vision to be important. The expectation for excellent quality of vision is a required consideration for continuous improvements in IOL technology. Of the responding surgeons, $43.1 \%$ cited glasses independence as important while $25.4 \%$ did not feel it was important. Attitudes about glasses independence may be influenced by personal experience and experience with patients. Risk tolerance may also influence surgeons' interest in being glasses free. The analysis revealed that over $50 \%$ of surgeons consider themselves risk averse while only $18.0 \%$ consider themselves to be risk takers. The factors that influence attitudes concerning risk were beyond the purview of this study.

Patients often ask their surgeons to recommend the IOL they would choose for their own surgery. It is interesting to note that, despite the fact that $>60 \%$ of surgeons implant presbyopia-correcting IOL, $>60 \%$ of surgeons would choose a monofocal lens for themselves. In the setting of corneal astigmatism $>1.25 \mathrm{D}, 60.3 \%$ of surgeons would prefer to have a toric monofocal for their own surgery. The second most popular choice overall was the toric diffractive echelette to correct corneal astigmatism (14.0\%). The diffractive echelette IOL was a popular choice in the presence of corneal astigmatism and minimal astigmatism even though it is a relatively new presbyopia-correcting IOL option. Monofocal options were the most popular surgeon preference whether or not astigmatism was present, suggesting that quality of vision is more important to the respondents than glasses independence.

A primary aim of this study was to determine whether personal IOL selection was influenced by demographics, experience, or personal characteristics. Associations between surgeon IOL choice and nine variables were analyzed. Surgeons who implanted $>50$ multifocal IOL or accommodative IOL were 2 times more likely to select a presbyopia-correcting IOL for themselves. This suggests that surgical experience in patient care influences an ophthalmologist's decision-making for his or her own cataract surgery. Surgeons who value glasses independence are two times more likely to select a presbyopia-correcting IOL. One would expect that a surgeon with experience implanting presbyopia-correcting IOLs who is seeking glasses independence would be likely to select one for themselves and the data support this premise. In the setting of corneal astigmatism $>1.25 \mathrm{D}$, surgeons who implanted $>50$ accommodative IOL and value glasses independence are three times and four times more likely to choose a presbyopiacorrecting IOL option, respectively. This data suggest that surgeons who have less experience with a particular technology are less likely to select it for themselves.

FLACS is an additional option available to the contemporary cataract surgeon. There has been considerable discussion regarding the benefit of this technology and whether it should be used on a regular basis [2-4]. The data from
Table 2 demonstrates that surgeons' personal decisionmaking is related to their personal experience. Of the surgeons who use the femtosecond laser more than one time per week, $55.9 \%$ said they would want it used for themselves, while only $3.5 \%$ of those not certified said they would want the laser used for themselves. A future study could explore in greater depth surgeons' attitudes about FLACS.

In addition to studying surgeons' personal IOL choices, the data revealed information regarding IOL preferences for patients. A direct comparison of surgeons' personal IOL choices and choices for patients in the setting of absent corneal astigmatism was not possible, but there was data regarding presbyopia-correcting IOL selection for patients. Over $65 \%$ of ophthalmologists chose a multifocal or diffractive echelette IOL for patients when using a presbyopiacorrecting lens. This data also includes surgeons who said they do not perform cataract surgery (16.0\%). In effect, the choice to use multifocal or diffractive echelette IOL for patients when using a presbyopia-correcting lens is actually higher if one considers only surgeons who perform cataract surgery. The explanation for these preferences was not studied in this survey. Additional study would be needed to further understand which factors were most important in making this decision. In a direct comparison of surgeon personal IOL choice and IOL choice for the patient in the setting of astigmatism (Fig. 3), it is noteworthy that in both instances $>60 \%$ of ophthalmologists indicated preference for the toric monofocal. It is possible the ease of insertion, the high quality of vision correction, and the high probability of reducing the need for glasses at a distance in the setting of astigmatism explains this strong preference for the toric monofocal.

A limitation of this study is that it was not designed to specifically compare personal IOL choice versus IOL choice for patients. The data demonstrate that a surgeon's past experience with a particular IOL or femtosecond laser may influence their choice for their own cataract surgery. The study does not address whether surgeons would be unwilling to personally have a specific IOL used on patients used on themselves. An additional limitation was that the number of surgeons who had actually had cataract surgery already and the number of hyperopes was very low; we were therefore unable to find significant data that related to those surgeons' IOL choices. After we had received approximately 150 survey responses, we added a question to the survey inquiring about the respondents' gender leaving half the respondents without gender data. For the purpose of this report, we decided to consider data we had received from all 328 respondents. Finally, we were unable to determine a response rate to our survey link because we had no way of realistically knowing how many ophthalmologists actually received the email link. This can potentially limit the generalizability of our data, but our demographic data suggest that our respondents were a well-balanced, diverse group of ophthalmologists. 
In conclusion, ophthalmologists are risk averse and value quality of vision. Surgeons who have more experience implanting presbyopia-correcting IOL and value glasses independence are more likely to select a presbyopia-correcting IOL for their own cataract surgery. Surgeons with more experience with FLACS are more likely to choose it for their own surgery. FLACS is being performed by a minority of surgeons and the majority are not interested in using it for their own surgery. The continued advances in cataract surgery technologies present the contemporary ophthalmologist and cataract patient with many options. It is important for cataract surgeons to consider the factors that influence their opinions with regard to these surgical options in order to objectively help their patients decide on the most suitable option.

\section{Summary}

\section{What was known before}

- Ophthalmologists choose among various IOLs and surgical modalities such as FLACS when preparing for a patient's cataract surgery.

- The preferences ophthalmologists have for their own cataract surgery have not been studied.

\section{What this study adds}

- Surgical experience does impact how ophthalmologists would choose to have their own cataract surgery completed.

- FLACS use is highly variable among ophthalmologists.
Funding This work received an unrestricted grant from Research to Prevent Blindness Inc., New York, NY, USA.

\section{Compliance with ethical standards}

Conflict of interest The authors declare that they have no conflict of interest.

Publisher's note: Springer Nature remains neutral with regard to jurisdictional claims in published maps and institutional affiliations.

\section{References}

1. Janssen SJ, Teunis T, Guitton TG, \& Ring D, Science of Variation Group. Do surgeons treat their patients like they would treat themselves? Clin Orthop Rel Res. 2015;473:3564-72.

2. Menapace RM, Dick HB. Femtosecond laser in cataract surgery. A critical appraisal. Ophthalmologe. 2014;111:624-37.

3. Grewal DS, Dalal RR, Jun S, Chou J, \& Basti S. Impact of the learning curve on intraoperative surgical time in femtosecond laser-assisted cataract surgery. J Refract Surg. 2016;32:311-7.

4. Roensch MA, Charton JW, Blomquist PH, Aggarwal NK, McCulley JP. Resident experience with toric and multifocal intraocular lenses in a public county hospital system. J Cataract Refract Surg. 2012;38:793-8.

5. Tang W, Zhuang S, Liu G. Comparison of visual function after multifocal and accommodative IOL implantation. Eye Sci. 2014;29:95-9.

6. Pepose JA, Burke J \& Qazi MA. Benefits and barriers of accommodating intraocular lenses. Curr Opin Ophthalmol. 2017;28:3-8.

7. Kretz FTA, Bastelica A, Carreras H, Ferreira T, Müller M, \& Gerl M, et al. Clinical outcomes and surgeon assessment after implantation of a new diffractive multifocal toric intraocular lens. Br J Ophthalmol. 2015;99:405-11.

8. Cochner B, Concerto Study Group. Clinical outcomes of a new extended range of vision intraocular lens: International Multicenter Concerto Study. J Cataract Refract Surg. 2016 Sep;42:1268-75. 